\title{
Design and Optimization of Electromagnetic Transmitter
}

\author{
Lili Li ${ }^{1,2, ~ a, ~ Q i a n g ~ F a n g ~}{ }^{2, ~ a}$ and Yulong Wang ${ }^{1,2, a}$
}

${ }^{1}$ Key Laboratory of Engineering Dielectrics and Its Application, Ministry of Education, Harbin University of Science and Technology, Harbin 150080, China;

${ }^{2}$ College of Rongcheng, Harbin University of Science and Technology, Rongcheng 264300, China.

ahlgrongchenglll@163.com

Keywords: Electromagnetic transmitter, optimization, nested projectile, pulse powers, simulation.

\begin{abstract}
Sensors, a variety of pulse powers and nested projectiles were used to improve speed and efficiency of electromagnetic emitter. The influence of emission voltage, coil number and distance triggered on exit velocity of projectile was analyzed by experiments and simulations. The results show that speed of projectile in the new electromagnetic emitter is increased by $3.4 \%$ compared with the traditional one when the emission voltage is the same. When the number of coils is 90 , distance triggered is $15 \mathrm{~mm}$ and time of capacitor discharge is among $2-4 \mathrm{~ms}$, exit velocity of projectile is up to $32.4 \mathrm{~m} / \mathrm{s}$. The electromagnetic emitter is optimized by acceleration of nested projectile with magnetic resistance emitters and induction emitters to improve efficiency of energy. It can be confirmed that the significant impact will be formed in the future.
\end{abstract}

\section{Introduction}

With the rapid development of modern science and technology, technology of electromagnetic emitter was concerned gradually [1]. Birkeland in Norway proposed the concept of electromagnetic emission at first, and it was achieved by Australian R. A. Marshall that the electromagnetic gun which has $5 \mathrm{~m}$ long track accelerated $3.3 \mathrm{~g}$ polycarbonate projectile to $5.9 \mathrm{~km} / \mathrm{s}$, so that the feasibility of electromagnetic emission was proved, and the research on electromagnetic emission enters a new stage [2].Currently, the electromagnetic emitter has been applied in real life, and achieved works that the chemical propellant emitter can't accomplish, and it has a great impact on country's energy, transportation, defense and space planning.

A large number of papers were written mainly on theories of powers, coils and projectile, and so on, in electromagnetic emissions [3], but few researchers studied on driving force and operability of transmitters. In this article, theories of magnetic resistance emitters and induction emitters are combined to optimize structures of electromagnetic emitter and projectile, and then a mixed type of high efficiency electromagnetic launcher is proposed, which lays the foundation for a wide range of applications in the future [4].

\section{Basic Theory of Single-stage Electromagnetic Transmitter}

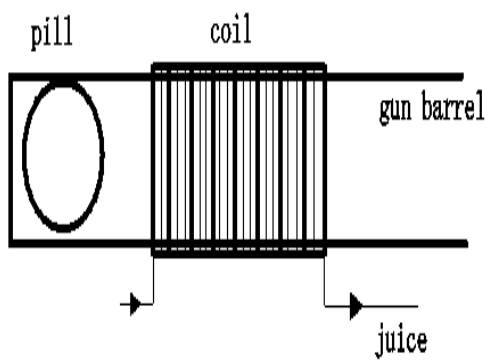

(a) Block diagram

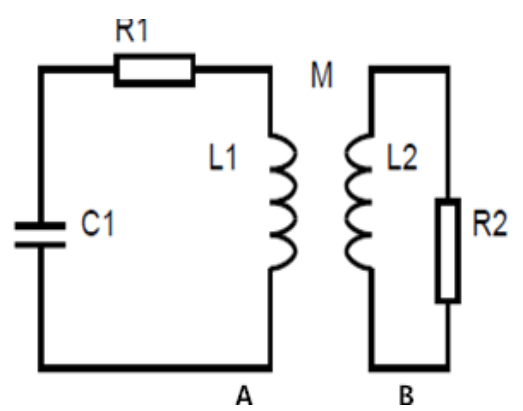

(b) Schematic

Fig. 1 Block diagram and schematic of single-stage electromagnetic emission

The magnetic field in electromagnetic launcher is generated by the pulse power or AC current, and emits projectile with coils or projectile with magnetic materials [5], the structure of electromagnetic 
launcher is shown in Figure 1(a). Electromagnetic launchers use coupling magnetic field to drive projectile moving with Lorentz force, which is formed in projectile and driving coil, as shown in the schematic diagram shown in figure 1(b).

In figure 1(b), the capacitor is used as pulse power, supposing that the magnetomotive force is proportional to coil numbers $N$ and coil current $i$, if the coil reluctance is $R_{m}$, the magnetic flux is $\Phi$, and $\Phi$ is proportional to $N \times i$, the formula (1) can be obtained [6].

$$
W_{m}=\int_{0}^{\Phi} \operatorname{Nid} \Phi=\frac{1}{2} \Phi^{2} R_{m}
$$

When the length and the cross section of magnetic circuit respectively are $L_{m}$ and $A_{m}$, the magnetic circuit reluctance is shown as formula (2).

$$
R_{m}=\frac{L_{m}}{\mu_{r} \mu_{0} A_{m}}
$$

Since the projectile is a ferromagnetic material, that is $\mu_{\text {rprojectile }} \gg \mu_{\text {rair }}$, the magnetic reluctance within coils is almost entirely generated by gaps $Q$ between the magnetic circuit coil and projectile, and magnetic lines pass through the gap $Q$ twice which is between launch tube and projectile, $L_{m} \approx 2 Q$. Assuming that the center of the projectile is coincident with the center of the drive coil, projectile diameter is $d_{m}$, projectile length is $L$, and $\varepsilon_{\text {rair }}=1$, the magnetic reluctance is shown as formula (3) [7].

$$
R_{m}=\frac{4 g}{\mu_{r} \pi d_{m} L}
$$

The diameter of emission coils is $d_{n}$, the drive coils length is about twice polar distance. Ignoring impacts of other factors, the energy stored $W_{n}$ is differential of movement distance $x$ in the middle axis is the thrust to the Projectiles, as shown in equation (4).

$$
F=\frac{d w_{n}}{d x}=\frac{1}{2} \Phi^{2} \frac{d R_{n}}{d x} \approx(N i)^{2} \frac{4 Q}{\mu_{r} \pi d_{n} L^{2}}
$$

Equation (4) shows that $F$ is $\infty$, when $N$ is $\infty$. But $N$ can't be infinite in the practical application of a single-stage pulse transmitter, and projectile reaching center of coils would be subjected to backward force to impede its movement.

\section{Design of New Electromagnetic Transmitter}

Main Design. Structure diagram of single-stage electromagnetic transmitter is shown in figure 2. The key in launch system is structure of projectile, projectile is built body with a sleeve-type structure to meet reluctance and induction type transmitter requirements, and it is shown in figure 3 . Transmitter tube is the PVC pipe which is twined by enameled wires, and transmitter sensors are installed respectively in the first end and the midpoint of tube to determine the position of projectile.

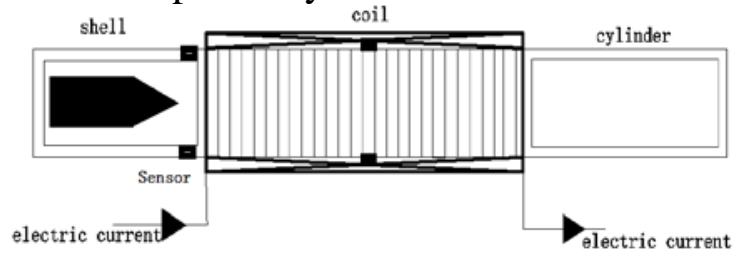

Fig. 2 electromagnetic transmitter's structure

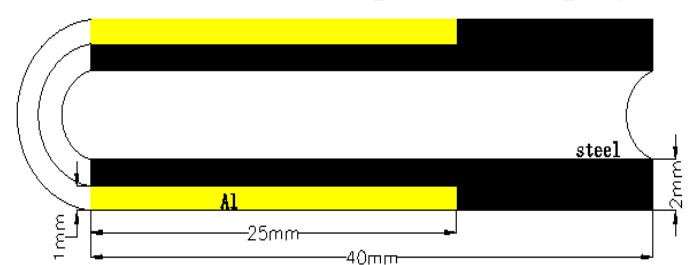

Fig. 3 new projectile's structure and parameters

Circuit Design of Transmitter. In this paper, the discharge circuit is improved mainly, and as shown in figure 5 . It is shown in figure 4 that single-pulse power increased to two pulse powers in the new electromagnetic emitter. 


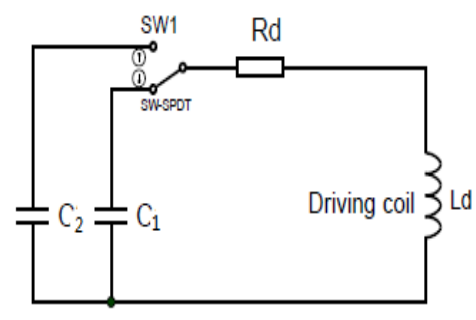

Fig. 4 Circuit design of new electromagnetic transmitter

The soft switch is closed when the first sensor detect projectile in the process of projectile motion, so that the capacitor $C_{1}$ storing energy releases pulse current, projectile is only accelerated because of projectile structure in magnetic resistance emitter without vortex. When projectile moves to midpoint of coils, the second sensor controls soft switching movement from $C_{1}$ to $C_{2}$, so that another pulse current is applied to coils again, there is larger eddy current in the tail of the projectile with aluminum, and the induction emission is realized [8]. In summary, the design takes advantage of reluctance electromagnetic transmitters and inductive electromagnetic transmitters by $C_{1}$ and $C_{2}$.

\section{Analysis of Experimental Tests and Simulation Results}

Influence of Emission Voltage on Exit Velocity of Projectile. The relationship between test voltage and exit velocity of projectile in electromagnetic launcher optimized and traditional launcher were shown in figure 6. It can be seen clearly in figure 5 that projectile's exit velocity increases with the increase of emission voltage, regardless of the structure of electromagnetic launcher, and that the correlation coefficient between voltage and exit velocity is approximately linear through test data fitting, which was 0.974 . The exit velocity of projectile is significantly higher in new electromagnetic launcher and the speed increase by 3.4\%, when voltage was the same. In summary, the projectile's speed and the working efficiency are obviously improved in new electromagnetic launcher.

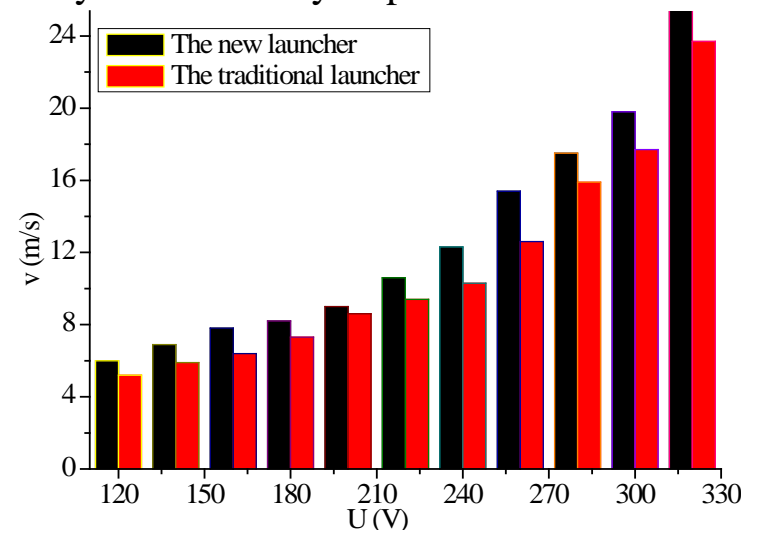

Fig. 5 The relationship between test voltage and exit velocity of projectile,

Influence of Trigger Distance on Exit Velocity of Projectile. The initial position has a great influence on transmission system, and the trigger distance of transmitting coils is less than half of distance generally. The relationship between trigger distance and exit velocity of projectile was shown in figure 6 , and it was assumed that the drive coil end is zero reference point of trigger position. It can be seen in figure 6 that exit velocity of projectile appeared the trend which is first increasing and then decreasing and exit velocity of projectile emerge from maximum value when the trigger distance was $15 \mathrm{~mm}$ with increasing of trigger distance. The main reason is that the trigger depth is too deep and decaying current will be produced a force against it moving when the trigger distance is shorter, so that exit velocity was reducing. Projectile's electromagnetic force is smaller beyond scope of coils when the trigger distance was longer, and it results in exit velocity lower. Therefore, the transmission efficiency is the highest only when the trigger distance is appropriate. 


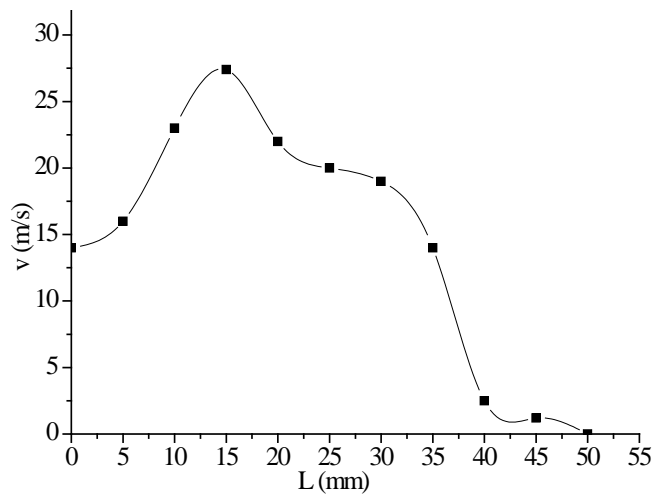

Fig. 6 Curve of exit velocity with trigger distance

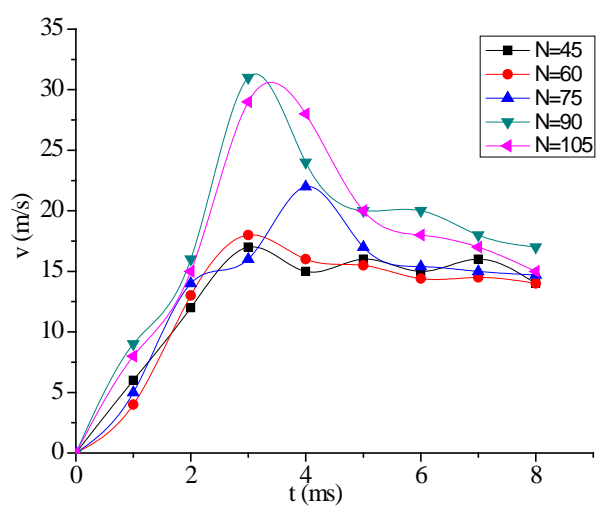

Fig. 7 Curve of exit velocity with coils number

Influence of Driving Coils Number and Discharge Time on Exit Velocity of Projectile. The relationship between discharge time and exit velocity of projectile with changing of driving coils number was shown in figure 7 . The relationship between discharge time and exit velocity of projectile with changing of driving coils number. As can be seen in figure 8, projectile's exit velocity increased first and then reduced with the increase of discharge time, and the maximum of exit velocity occurred within 2-4ms. The maximum value of projectile's exit velocity increased first and then reduced with the increase of the number of $\mathrm{N}$, and the maximum value is $32.4 \mathrm{~m} / \mathrm{s}$ when $\mathrm{N}$ is 90 . Reasons for phenomenon are that the electromagnetic force generated by drive coils is small when $\mathrm{N}$ is smaller and less impact on the speed of increase, and that the electromagnetic force increases with the increase of $\mathrm{N}$, but the corresponding discharge time is prolonged, so that projectile's movement of is also hindered. Therefore, the maximum value of exit velocity is maximum, only when $\mathrm{N}=90$.

\section{Conclusion}

The new electromagnetic launcher was built by changing projectile structure and installing sensors to increase efficiency and can accelerate projectile in the entire driving coil. Following conclusions through experiments and simulations were obtained:

(1) When transmitter voltage is the same, discharge time of capacitor is controlled by sensors, energy loss is reduced and energy utilization ratio is improved, so that projectile exit velocity in the new electromagnetic emitter is improved by 3.4\% compared with the traditional emitter.

(2) When the projectile trigger distance is about $15 \mathrm{~mm}$ in the driving coil, resistance force which is produced by attenuation current is smaller and propulsive force applied on projectile is larger, in result that the projectile's largest export speed is about $27.3 \mathrm{~m} / \mathrm{s}$.

(3) When projectile trigger distance is about $15 \mathrm{~mm}$ and number of driving coils is 90 , resistance force which is produced by attenuation current is the smallest and propulsive force applied on projectile is the largest, so that the projectile's largest export speed has the maximum which is about $32.4 \mathrm{~m} / \mathrm{s}$.

\section{Literature References}

[1] HOU Yan-pan, LIU Zhen-xiang, YANG Li-jia, et al, Dynamic Simulation and Experiment of the Reluctance Coil Launcher, Journal of Sichuan Ordnance. 34 (2013) 43-45.

[2] HUANG Hai, GAO Jun-shan, LI Wen-long, DSP-Control of the Electromagnetic Launcher, Techniques of Automation and Application. 26(2007) 92-96.

[3] DAI Guang-zhen, JIANG Liu, WANG Feng-sui, et al, Research and Design of Electromagnetic Launcher of the two Stage coil based on AVR Microcontroller, Journal of Chifeng University (NATURAL SCIENCE EDITION). 31 (2015) 4-8.

[4] LI Xiang-cheng, Liu Zhen-xiang, HOU Yang-pan, et al, Analysis of Back Current in Coil Electromagnetic Launchers and the Effect Acting on the Launching System. 35 (2014) 59-63. 
[5] CUI Peng, Research on the New Electromagnetic Launch Technology (MS. National University of Defense technology, China 2009)

[6] SUN Zhong-he, WU Bo, GAO Jun-shan, Research on Simulation and Test of the Pint-sized Single-stage Electromagnetic Launcher, Journal of Harbin University of Science and Technology. 14 (2009) 34-36.

[7] FEI Fui-cong, LI Yuan-cheng, TANG Yong, et al, Fabrication and Study of Multi-stage Magnetic resistive Electromagnetic Gun based on Sensor Control, Physics and Engineering, 23 (2013) 25-28.

[8] OUYANG Jian-ming, LIU Zhen-xiang, YANG Li-jia, et al, Dynamic Numerical Emulation of New Electromagnetic Launcher , MICROMOTORS, 44 (2011) 25-27. 